\title{
Father's knowledge and attitude towards breast feeding
}

\author{
K. Abhinaya ${ }^{1}$, T. S. Arunprasath ${ }^{2}$, L. N. Padmasani ${ }^{3}$ \\ ${ }^{1}$ Ms. Abhinaya, Final year MBBS, ${ }^{2}$ Dr. T. S. Arunprasath, Assistant Professor in Pediatrics, ${ }^{3}$ Dr. L. N. Padmasani, \\ Professor of Pediatrics, authors are affiliated with Sri Ramachandra Medical College and Research Institute, Porur, \\ Chennai, India.
}

Address for Correspondence: Dr. T. S. Arunprasath, Email: drtsarunprasath@yahoo.com

\begin{abstract}
Introduction: A father's knowledge and attitude towards breastfeeding are very important for the success of breastfeeding practices. There is no uniform practice of including them in the education sessions in our country. Objectives: To assess the knowledge and attitude towards breastfeeding among fathers of infants aged 6 months to 2 years attending well baby clinic. Material and Method: A cross sectional study with convenient sampling was conducted on 93 fathers. A self-administered, pretested and pre-validated questionnaire with regards to breastfeeding practices was then distributed among the fathers. Results: 93 respondents completed this study. 70 fathers $(75.3 \%)$ had babies exclusively breast fed between 4-6 months of age. 54 fathers $(58.1 \%)$ had knowledge about breastfeeding before baby's birth and $68(73.1 \%)$ were keen to get more information after baby's birth. 90 fathers $(96.8 \%)$ felt that they got required information, but only $34(36.6 . \%)$ had received education from health care providers. Knowledge regarding exclusive breastfeeding $(n=84)$, breast milk superiority, $(n=86)$, emotional bonding created by breastfeeding $(n=70)$ was adequate in most. Father's age, education status, occupation, family type had no influence on knowledge or attitude. Father's whose child was born by caesarean section or had co morbidities in neonatal period had better knowledge and positive attitude. Fathers with better knowledge had positive attitude towards breastfeeding, but this had no influence on duration of exclusive breastfeeding. Conclusion: Father's knowledge was positively affected by health care exposure and education. Fathers with good knowledge had positive attitude towards breast feeding. Hence they should be included in discussions regarding breastfeeding in antenatal counselling.
\end{abstract}

Keywords: knowledge, Fathers, Breastfeeding

\section{Introduction}

The World Health Organization [WHO] recommends exclusive breastfeeding [EBF] till six months of age [1]. In spite of many initiatives to promote $\mathrm{EBF}$, the rates continue to be low in India [2]. The influences of factors like maternal education, socioeconomic status and antenatal services have been studied in detail. [3, 4, 5]. However, a woman's decision is often influenced by her partner's attitude [6]. There is very little data available about Indian fathers' knowledge and attitude towards breastfeeding.

\section{Methodology}

A cross sectional, observational study was conducted in

Manuscript received $25^{\text {th }}$ September 2016

Reviewed: $7^{\text {th }}$ October 2016

Author Corrected: $19^{\text {th }}$ October 2016

Accepted for Publication $31^{\text {st }}$ October 2016
May - June 2016, as a part of MBBS Undergraduate Summer research fellowship program in our university. Fathers of children aged 6 months to 2 years, coming for well-baby check-up were interviewed with a structured pre-validated questionnaire.

Tools: KAP questionnaire: The questionnaire had five sections: Section 1 on baby's and parents sociodemographic details, Section 2 on baby's details like birth and feeding details, Section 3 to evaluate fathers knowledge about breast feeding and Section 4 to evaluate attitude of fathers towards breastfeeding.

A 5 point Likert scale was applied [strongly agree $=+2$, agree $=+1$, neutral $=0$ disagree $=-1$, and strongly disagree $=-2$ ] for the knowledge questions valued as positive and [strongly agree $=-2$, agree $=-1$, neutral $=0$ 
disagree $=+1$, and strongly disagree $=+2$ ] for the knowledge questions valued as negative. Similar scales were applied for the statements used to test attitude.

The total score was calculated and was transformed into mean 'percent score' by dividing the score with possible maximum score and multiplied by 100 . Scores for Knowledge was categorized as poor [0-<=30\%], moderate $[>30 \%-<=70 \%]$ and high $[>70 \%]$. Scores for attitude was categorized as negative $[0-<=30 \%]$, neutral $[>30 \%-<=70 \%]$ and positive $[>70 \%]$.
The study was done with the approval of the Institution's Ethics Committee and informed consent of parents.

Analysis: Descriptive analysis was done to analyse the socio-demographic data and Pearson Chi square test analysis was used to compare the knowledge score with attitude score and to compare the knowledge and attitude scores with other variables.

\section{Results}

A total of 93 respondents completed this study. The demographic details of the participants and their baby details is shown in Table 1-3. Majority babies had been exclusively breast fed for 4-6months of age. [n=70,75.3\%]

The mean knowledge score was 11.247 [SD=6.79] and the mean attitude score was 16.151[SD=7.52].

Table-1: Demographic variables of fathers.

\begin{tabular}{|c|c|c|}
\hline & Number & Percent \\
\hline \multicolumn{3}{|c|}{ Age group } \\
\hline 21 to 30 & 37 & 39.8 \\
\hline $31-40$ & 55 & 59.1 \\
\hline more than 40 & 1 & 1.1 \\
\hline \multicolumn{3}{|c|}{ Education } \\
\hline Profession & 8 & 8.6 \\
\hline Graduate & 44 & 47.3 \\
\hline Post High School Diploma & 9 & 9.7 \\
\hline High School & 24 & 25.8 \\
\hline Middle School & 5 & 5.4 \\
\hline Primary School & 3 & 3.2 \\
\hline \multicolumn{3}{|l|}{ Working status } \\
\hline Profession & 16 & 17.2 \\
\hline Semi-profession & 25 & 26.9 \\
\hline Clerical/shop owner / farmer & 4 & 4.3 \\
\hline Skilled worker & 27 & 29.0 \\
\hline Semi-skilled worker & 15 & 16.1 \\
\hline Unskilled worker & 6 & 6.5 \\
\hline \multicolumn{3}{|c|}{ Family type } \\
\hline Nuclear & 44 & 47.3 \\
\hline Joint & 49 & 52.7 \\
\hline \multicolumn{3}{|c|}{ Residence } \\
\hline Hut & 1 & 1.1 \\
\hline Flat & 26 & 28.0 \\
\hline Independent house & 66 & 71.0 \\
\hline
\end{tabular}


Table 2: Demographic variables of baby details.

\begin{tabular}{|c|c|c|}
\hline Baby details & Number & Percent \\
\hline \multicolumn{3}{|c|}{ Sex } \\
\hline Male & 45 & 48.4 \\
\hline Female & 48 & 51.6 \\
\hline \multicolumn{3}{|c|}{ Birth Order } \\
\hline $1^{\text {st }}$ born & 62 & 66.7 \\
\hline $2^{\text {nd }}$ born & 24 & 52.8 \\
\hline $3^{\text {rd }}$ born & 6 & 6.5 \\
\hline $4^{\text {th }}$ born & 0 & 0 \\
\hline $5^{\text {th }}$ born & 1 & 1.1 \\
\hline \multicolumn{3}{|c|}{ Gestation age } \\
\hline Term & 83 & 89.2 \\
\hline Pre-term & 9 & 9.7 \\
\hline Post-term & 1 & 1.1 \\
\hline \multicolumn{3}{|c|}{ Birth weight } \\
\hline$<2.5 \mathrm{~kg}$ & 28 & 30.1 \\
\hline $2.5-4 \mathrm{~kg}$ & 64 & 68.8 \\
\hline$>4 \mathrm{~kg}$ & 1 & 1.1 \\
\hline \multicolumn{3}{|c|}{ Nicu stay in hours } \\
\hline$<24$ & 89 & 95.7 \\
\hline $24-72$ & 4 & 4.3 \\
\hline \multicolumn{3}{|c|}{ Co Morbidities } \\
\hline Absent & 85 & 91.4 \\
\hline Present & 8 & 8.6 \\
\hline \multicolumn{3}{|c|}{ Mode Of Delivery } \\
\hline Vaginal & 53 & 57.0 \\
\hline Assisted & 4 & 4.3 \\
\hline Lscs & 36 & 38.7 \\
\hline
\end{tabular}

54 fathers [58.1\%] had knowledge about breastfeeding before baby was born and 68 [73.1\%] were keen to get more information after the baby was born.

90 fathers [96.8\%] felt that they got required information about breastfeeding, but only 34 [36.6. \%] of them had received education from health care providers. Knowledge regarding recommended duration of exclusive breast feeding [n=84, $90.3 \%$, breast milk superiority over formula/cow's milk [ $\mathrm{n}=86,92.5 \%]$ and promotion of emotional bonding by breastfeeding $[\mathrm{n}=70,75.2 \%]$ was adequate in most fathers.

However, the knowledge that breast feeding reduces postpartum depression in mother [n=27, 29\%] and weight gain $[\mathrm{n}=15,16.1 \%]$ Also, knowledge that after LSCS also, mothers can produce enough milk [n=31, 33.3\%] was inadequate. Majority fathers $[\mathrm{n}=51]$ felt that breastfeeding should be continued for more than a year. 
Fathers in the age group of 31-40, had more knowledge than those in the age group of 21-30 but the difference was not statistically significant.

Attitude was neutral in the fathers of 21-30 age group, [ $n=18]$ and was positive in 31-40 age group, [n=26] but again the difference was not statistically significant.

Table 3: Feeding pattern of subject.

\begin{tabular}{|c|c|c|}
\hline Feeding details & Number & Percent \\
\hline \multicolumn{3}{|c|}{ Time of initiation of breastfeeding } \\
\hline$<=1$ hour & 34 & 36.6 \\
\hline$>1-4$ hours & 29 & 31.2 \\
\hline$>4-24$ hours & 15 & 16.1 \\
\hline$>24$ hours & 15 & 16.1 \\
\hline \multicolumn{3}{|c|}{ Prelacteal feeds given } \\
\hline No & 79 & 84.9 \\
\hline Yes & 14 & 15.1 \\
\hline \multicolumn{3}{|c|}{ Duration of exclusive breastfeeding } \\
\hline$<=4$ months & 17 & 18.2 \\
\hline $4-6$ months & 70 & 75.3 \\
\hline$>6$ months & 6 & 6.5 \\
\hline \multicolumn{3}{|c|}{ Early initiation of complimentary feeds } \\
\hline Not enough milk & 51 & 55.4 \\
\hline Inadequate milk & 23 & 25.0 \\
\hline Family influences & 17 & 18.5 \\
\hline Others & 1 & 1.1 \\
\hline \multicolumn{3}{|c|}{ Duration of stay in maternal home } \\
\hline$<=4$ months & 60 & 64.5 \\
\hline $4-6$ months & 25 & 26.9 \\
\hline$>6$ months & 8 & 8.6 \\
\hline
\end{tabular}

Fathers educational and work status and the family type did not have any influence on knowledge or attitude of breast feeding practices.

Birth order of the child had a negative influence, with knowledge and attitude declining with increasing birth order. Fathers whose child had co morbidities in the neonatal period or hospitalization in the neonatal period had more knowledge and a positive attitude. When the child was delivered by Caesarean section, fathers had good knowledge and positive attitude [Table 4]. 
Table-4: Fathers knowledge score and attitude score in comparison with variables.

\begin{tabular}{|c|c|c|c|c|c|}
\hline \multirow[t]{2}{*}{ Variable } & \multicolumn{3}{|c|}{ Knowledge score in percent } & \multirow[t]{2}{*}{ Total } & \multirow[t]{2}{*}{ Significance } \\
\hline & less than 30 & $30-70$ & $>70$ & & \\
\hline \multicolumn{6}{|c|}{ Age } \\
\hline 21 to 30 & 22 & 12 & 3 & 37 & \multirow[b]{3}{*}{.047} \\
\hline $31-40$ & 18 & 35 & 2 & 55 & \\
\hline more than 40 & 0 & 1 & 0 & 1 & \\
\hline \multicolumn{6}{|c|}{ Education } \\
\hline Profession & 5 & 3 & 0 & 8 & \multirow{6}{*}{.157} \\
\hline Graduate & 17 & 24 & 3 & 44 & \\
\hline $\begin{array}{l}\text { Post High School } \\
\text { Diploma }\end{array}$ & 6 & 3 & 0 & 9 & \\
\hline High School & 6 & 16 & 2 & 24 & \\
\hline Middle School & 5 & 0 & 0 & 5 & \\
\hline Primary School & 1 & 2 & 0 & 3 & \\
\hline \multicolumn{6}{|c|}{ Working status } \\
\hline Profession & 8 & 7 & 1 & 16 & \multirow{6}{*}{.019} \\
\hline Semi-profession & 8 & 17 & 0 & 25 & \\
\hline $\begin{array}{l}\text { Clerical/shop } \\
\text { owner / farmer }\end{array}$ & 1 & 1 & 2 & 4 & \\
\hline Skilled worker & 13 & 12 & 2 & 27 & \\
\hline $\begin{array}{l}\text { Semi-skilled } \\
\text { worker }\end{array}$ & 7 & 8 & 0 & 15 & \\
\hline Unskilled worker & 3 & 3 & 0 & 6 & \\
\hline \multicolumn{6}{|c|}{ Family type } \\
\hline Nuclear & 20 & 21 & 3 & 44 & \multirow[t]{2}{*}{.711} \\
\hline Joint & 20 & 27 & 2 & 49 & \\
\hline \multicolumn{6}{|c|}{ Birth order } \\
\hline 1 & 23 & 34 & 5 & 62 & \multirow{4}{*}{.003} \\
\hline 2 & 17 & 7 & 0 & 24 & \\
\hline 3 & 0 & 6 & 0 & 6 & \\
\hline 5 & 0 & 1 & 0 & 1 & \\
\hline \multicolumn{6}{|c|}{ Co morbidities in baby } \\
\hline No & 38 & 42 & 5 & 85 & \multirow[b]{2}{*}{.525} \\
\hline Yes & 2 & 6 & 0 & 8 & \\
\hline \multicolumn{6}{|l|}{ mode of delivery } \\
\hline Vaginal & 26 & 25 & 2 & 53 & \multirow[b]{3}{*}{.115} \\
\hline Assisted & 2 & 2 & 0 & 4 & \\
\hline LSCS & 12 & 21 & 3 & 36 & \\
\hline
\end{tabular}




\begin{tabular}{|c|c|c|c|c|c|}
\hline \multirow[t]{2}{*}{ Variable } & \multicolumn{3}{|c|}{ Attitude score in percent } & \multirow[t]{2}{*}{ Total } & \multirow[t]{2}{*}{ Significance } \\
\hline & less than 30 & 30-70 & $>70$ & & \\
\hline \multicolumn{6}{|c|}{ Age } \\
\hline 21 to 30 & 8 & 18 & 11 & 37 & \multirow[b]{3}{*}{.348} \\
\hline $31-40$ & 7 & 22 & 26 & 55 & \\
\hline more than 40 & 0 & 1 & 0 & 1 & \\
\hline \multicolumn{6}{|c|}{ Education } \\
\hline Profession & 2 & 3 & 3 & 8 & \multirow{6}{*}{.629} \\
\hline Graduate & 6 & 21 & 17 & 44 & \\
\hline $\begin{array}{c}\text { Post High } \\
\text { School Diploma }\end{array}$ & 3 & 4 & 2 & 9 & \\
\hline High School & 2 & 10 & 12 & 24 & \\
\hline Middle School & 2 & 1 & 2 & 5 & \\
\hline Primary School & 2 & 1 & 2 & 5 & \\
\hline \multicolumn{6}{|c|}{ Working status } \\
\hline Profession & 3 & 8 & 5 & 16 & \multirow[t]{3}{*}{.932} \\
\hline Semi-profession & 2 & 12 & 11 & 25 & \\
\hline $\begin{array}{c}\text { Clerical/shop } \\
\text { owner / farmer }\end{array}$ & 1 & 2 & 1 & 4 & \\
\hline Skilled worker & 4 & 10 & 13 & 27 & \\
\hline $\begin{array}{l}\text { Semi-skilled } \\
\text { worker }\end{array}$ & 4 & 6 & 5 & 15 & \\
\hline $\begin{array}{l}\text { Unskilled } \\
\text { worker }\end{array}$ & 1 & 3 & 2 & 6 & \\
\hline \multicolumn{6}{|l|}{ Family type } \\
\hline Nuclear & 7 & 20 & 17 & 44 & \multirow[t]{2}{*}{.968} \\
\hline Joint & 8 & 21 & 20 & 49 & \\
\hline \multicolumn{6}{|l|}{ birth order } \\
\hline 1 & 9 & 28 & 25 & 62 & \multirow{4}{*}{.468} \\
\hline 2 & 6 & 11 & 7 & 24 & \\
\hline 3 & 0 & 2 & 4 & 6 & \\
\hline 5 & 0 & 0 & 1 & 1 & \\
\hline \multicolumn{6}{|l|}{$\begin{array}{l}\text { Co morbidities } \\
\text { in baby }\end{array}$} \\
\hline No & 15 & 38 & 32 & 85 & \multirow[b]{2}{*}{.107} \\
\hline Yes & 0 & 3 & 5 & 8 & \\
\hline \multicolumn{6}{|l|}{$\begin{array}{l}\text { Mode of } \\
\text { delivery }\end{array}$} \\
\hline Vaginal & 9 & 24 & 20 & 53 & \multirow[b]{3}{*}{.648} \\
\hline Assisted & 0 & 3 & 1 & 4 & \\
\hline LSCS & 6 & 14 & 16 & 36 & \\
\hline
\end{tabular}

Father's knowledge regarding breast feeding had no influence on the practice of giving prelacteal feeds or duration of exclusive breast feeding [Table 5]. 
Table-5: Father's knowledge score and attitude score influencing duration of exclusive breastfeeding

\begin{tabular}{|c|c|c|c|c|c|}
\hline \multirow{2}{*}{ Variable } & \multicolumn{2}{|c|}{ Duration of exclusive breastfeeding } & \multicolumn{2}{|c|}{ Total } & Significance \\
\cline { 2 - 5 } & $\begin{array}{c}\text { less than } \\
\text { 4months }\end{array}$ & 4-6months & $\begin{array}{c}\text { more than } \\
\text { 6months }\end{array}$ & \multicolumn{2}{|c|}{} \\
\hline \multicolumn{7}{|c|}{ Knowledge score in percent } \\
\hline less than 30 & 6 & 31 & 3 & 40 & \multirow{2}{*}{.451} \\
\hline $30-70$ & 9 & 37 & 2 & 5 & 5 \\
\hline$>70$ & 2 & 2 & 1 & & \\
\hline
\end{tabular}

\begin{tabular}{|c|c|c|c|c|c|}
\hline \multirow{2}{*}{ Variable } & \multicolumn{2}{|c|}{ Duration of exclusive breastfeeding } & \multicolumn{2}{c|}{ Total } & Significance \\
\cline { 2 - 5 } & $\begin{array}{c}\text { less than } \\
\text { 4months }\end{array}$ & 4-6months & $\begin{array}{c}\text { more than } \\
\text { 6months }\end{array}$ & \multicolumn{2}{|c|}{} \\
\hline \multicolumn{7}{|c|}{ Attitude score in percent } \\
\hline less than 30 & 1 & 13 & 1 & 41 & \multirow{2}{*}{.617} \\
\hline $30-70$ & 7 & 31 & 3 & 37 & \\
\hline$>70$ & 9 & 26 & 2 & & \\
\end{tabular}

Table-6: Knowledge affecting the attitude towards breast feeding

\begin{tabular}{|c|c|c|c|c|}
\hline & \multicolumn{3}{|c|}{ Knowledge percent score } \\
\hline $\begin{array}{c}\text { Attitude percent } \\
\text { score }\end{array}$ & less than 30 & $\mathbf{3 0 - 7 0}$ & $\mathbf{7 0}$ & Total \\
\hline less than 30 & 14 & 0 & 1 & 15 \\
\hline $30-70$ & 16 & 23 & 2 & 41 \\
\hline$>70$ & 10 & 25 & 2 & 37 \\
\hline Total & 40 & 48 & 5 & 93 \\
\hline
\end{tabular}

Significance $=.001$

Fathers with better knowledge had positive attitude towards breastfeeding and it was statistically significant [Table 6].

\section{Discussion}

Fathers in our study were keen to get information about breastfeeding, similar to fathers in other studies [7] but only half of them had adequate knowledge about breastfeeding before birth of baby. This may be because of limited involvement of fathers during antenatal checkup in our country. As reported in earlier studies from India, after child birth, only a few fathers got information about breastfeeding from healthcare professional [8]. As a routine, fathers are not involved in infant feeding education decisions in our setting.

As reported earlier, most fathers knew that breast milk was superior to formula [9] and that it increased emotional bonding between mother and baby [10]. In contrast to other studies, fathers education status had no influence on knowledge or attitude towards breast feeding [11] probably due to the influence of the family structure in our country. Neither nuclear nor joint family type had an influence on knowledge and attitude of the fathers in our study suggesting non involvement of fathers in infant feeding decisions in our setting.

As reported earlier, fathers in our study exhibited positive attitude towards breastfeeding, when they had a child delivered by Caesarean section or when the baby was hospitalized for comorbidities [12] probably due to exposure to health care professional and health education. Even fathers with moderate knowledge and positive attitude, could not influence the duration of exclusive breast feeding and this finding is reported by Karande [13], while study done by Susin [14] reports otherwise. This difference may be attributed to family dynamics in our setting and dominant role played by females in decision making strategies of infant feeding. Previous studies have shown that if fathers are educated and involved in infant feeding decisions making, the 
rates of exclusive breast feeding will definitely increase [15]. In our study fathers with good knowledge had a positive attitude towards breastfeeding even though they did not have antenatal classes or postnatal involvement in infant feeding

\section{Conclusion}

Father's knowledge was positively affected by health care exposure and education. Fathers with good knowledge had a positive attitude towards breast feeding. If fathers are educated by health care professionals the rates of exclusive breastfeeding will improve.

Funding: Nil, Conflict of interest: None initiated,

Permission from IRB: Yes

\section{Bibliography}

1. World Health Organization . Infant and young child feeding: model chapter for textbooks for medical students and allied health professionals. Geneva: World Health Organization; 2009

2. Chandhiok N, Singh KhJ, Sahu D, Singh L, Pandey A. Changes in exclusive breastfeeding practices and its determinants in India, 1992-2006: analysis of national survey data. Int Breastfeed J. 2015 Dec 29;10:34. doi: 10.1186/s13006-015-0059-0. eCollection 2015.

3. Tan K. Factors associated with exclusive breastfeeding among infants under six months of age in peninsular Malaysia. Int Breastfeed J. 2011;6(1):2. doi:10.1186/1746-4358-6-2

4. Agho KE, Dibley MJ, Odiase JI, Ogbonmwan SM. Determinants of exclusive breastfeeding in Nigeria. BMC Pregnancy Childbirth. 2011 Jan 11;11:2. doi: 10.1186/1471-2393-11-2.

5. Xu F, Binns C, Zheng S, Wang Y, Zhao Y, Lee A. Determinants of exclusive breastfeeding duration in Xinjiang, PR China. Asia Pac J Clin Nutr. 2007;16 (2):316-21.

6. Vaaler M, Castrucci B, Parks S, Clark J, Stagg J, Erickson T. Men's Attitudes Toward Breastfeeding: Findings from the 2007 Texas Behavioral Risk Factor
Surveillance System. Maternal and Child Health Journal. 2010;15(2):148-157. doi:10.1007/s10995-0100605-8

7. Brown A,Davies R . Fathers' experiences of supporting breastfeeding: challenges for breastfeeding promotion and education Matern Child Nutr. 2014 Oct; 10(4): 510-526. doi: 10.1111/mcn.12129

8. Bansal CP. Breastfeeding--why are we still failing? Indian Pediatr. 2013 Nov 8;50(11):993-4.

9. Pollock CA, Bustamante-Forest R, Giarratano G. Men of diverse cultures: knowledge and attitudes about breastfeeding. J Obstet Gynecol Neonatal Nurs. 2002 Nov-Dec;31(6):673-9.

10. Taşpınar A, Çoban A, Küçük M, Şirin A. Fathers' knowledge about and attitudes towards breast feeding in Manisa, Turkey. Midwifery. 2013 Jun;29(6):653-60. doi: 10.1016/j.midw.2012.06.005. Epub 2012 Jul 25.

11. Van Wagenen, S., Magnusson, B. and Neiger, B. Attitudes Toward Breastfeeding Among an Internet Panel of U.S. Males Aged 21-44. Maternal and Child Health Journal, 2015; 19(9):2020-2028. doi:10.1007/s 10995-015-1714-1

12. Sweet L, Darbyshire P. Fathers and breast feeding very-low-birthweight preterm babies. Midwifery. 2009 Oct;25(5):540-53. doi: 10.1016/j.midw.2007.09.001. Epub 2008 Jan 11.

13. Karande S, Perkar S.. Do fathers' attitudes support breastfeeding? A cross-sectional questionnaire-based study in Mumbai, India. Indian J Med Sci 2012;66(1): 30. doi:10.4103/0019-5359.110861

14. Susin, L., Giugliani, E., Kummer, S., Maciel, M., Simon, C. and da Silveira, L.Does Parental Breastfeeding Knowledge Increase Breastfeeding Rates?. Birth.1999; 26(3):149-156. doi:10.1046/j.1523536x.1999.00149.x

15. Maycock B, Binns CW, Dhaliwal S, Tohotoa J, Hauck Y, Burns S et al. Education and support for fathers improves breastfeeding rates: a randomized controlled trial. J Hum Lact. 2013;29(4):484-90. doi:10.1177/0890334413484387

\section{How to cite this article?}

K. Abhinaya, T. S. Arunprasath, L. N. Padmasani. Father's knowledge and attitude towards breast feeding. Int J Med Res Rev 2016;4(10):1778-1785.doi:10.17511/ijmrr. 2016.i10.12. 